myjournal manuscript No.

(will be inserted by the editor)

\title{
Polarization properties of subwavelength hole arrays consisting of
}

\section{rectangular holes}

\author{
Xi-Feng Ren, Pei Zhang, Guo-Ping Guo`, Yun-Feng Huang, Zhi-Wei Wang, Guang-Can Guo \\ Key Laboratory of Quantum Information, University of Science and Technology of China, Hefei 230026, People's Republic of \\ China
}

Received: date / Revised version: date

\begin{abstract}
Influence of hole shape on extraordinary optical transmission was investigated using hole arrays consisting of rectangular holes with different aspect ratio.

It was found that the transmission could be tuned continuously by rotating the hole array. Further more, a phase was generated in this process, and linear polarization states could be changed to elliptical polarization states. This phase was correlated with the aspect ratio of the holes. An intuitional model was presented to explain these results.

PACS numbers:78.66.Bz,73.20.MF, 71.36.+c
\end{abstract}

\section{1 introduction}

In metal films perforated with a periodic array of subwavelength apertures, it has long been observed that there is an unusually high optical transmission[1. It is

* E-mail: e-mail: :gpguo@ustc.edu.cn believed that metal surface plays a crucial role and the phenomenon is mediated by surface plasmon polaritons (SPPs) and there is a process of transforming photon to SPP and back to photon[2,3,4]. This phenomenon can be used in various applications, for example, sensors, optoelectronic device, etc $[5,6,7,8,9,10$. Polarization properties of nanohole arrays have been studied in many works 11, 12, 13. Recently, orbital angular momentum of photons was explored to investigate the spatial mode properties of surface plasmon assisted transmission [14,15]. It is also showed that entanglement of photon pairs can be preserved when they respectively travel through a hole array 15, 16, 17. Therefore, the macroscopic surface plasmon polarizations, a collective excitation wave involving typically $10^{10}$ free electrons propagating at the surface of conducting matter, have a true quantum nature. However, the increasing use of EOT requires further understanding of the phenomenon. 
The polarization of the incident light determines the mode of excited SPP which is also related to the periodic structure. For the manipulation of light at a subwavelength scale with periodic arrays of holes, two ingredients exist: shape and periodicity $[2,3,4,11,18,19,20$. Influence of unsymmetrical periodicity on EOT was discussed in 21]. Influence of the hole shape on EOT was also observed recently 18, 20, in which the authors mainly focused on the transmission spectra. In this work, we used rectangle hole arrays to investigate the influence of hole shape on the polarization properties of EOT. It is found that linear polarization states could be changed to elliptical polarization states and a phase could be added between two eigenmode directions. The phase was changed when the aspect ratio of the rectangle holes was varied. The hole array was also rotated in the plane perpendicular to the illuminate beam. The optical transmission was changed in this process. It strongly depended on the rotation angle, in other words, the angle between polarization of incident light and axis of hole array, as in the case with unsymmetrical hole array structure 21.

\section{2 experimental results and modeling}

\subsection{Relation between transmission efficiency and}

photon polarization

Fig. 1(a) is a scanning electron microscope picture of part of our hole arrays. The hole arrays are produced as follows: after subsequently evaporating a 3- $\mathrm{nm}$ tita- nium bonding layer and a $135-\mathrm{nm}$ gold layer onto a 0.5 $m m$-thick silica glass substrate, a focused ion beam etching system is used to produce rectangle holes $(100 \mathrm{~nm} \times$ $100 \mathrm{~nm}, 100 \mathrm{~nm} \times 150 \mathrm{~nm}, 100 \mathrm{~nm} \times 200 \mathrm{~nm}, 100 \mathrm{~nm} \times$ $300 \mathrm{~nm}$ respectively) arranged as a square lattice $(520 \mathrm{~nm}$ period). The area of the hole array is $10 \mu m \times 10 \mu m$.

Transmission spectra of the hole arrays were recorded by a silicon avalanche photodiode single photon counter couple with a spectrograph through a fiber. White light from a stabilized tungsten-halogen source passed though a single mode fiber and a polarizer (only vertical polarized light can pass), then illuminated the sample. The hole arrays were set between two lenses of $35 \mathrm{~mm}$ focal length, so that the light was normally incident on the hole array with a cross sectional diameter about $10 \mu \mathrm{m}$ and covered hundreds of holes. The light exiting from the hole array was launched into the spectrograph. The hole arrays were rotated anti-clockwise in the plane perpendicular to the illuminating light, as shown in Fig.

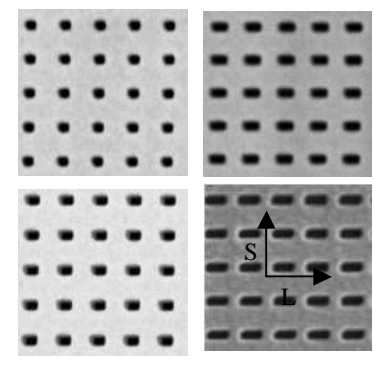

(a)

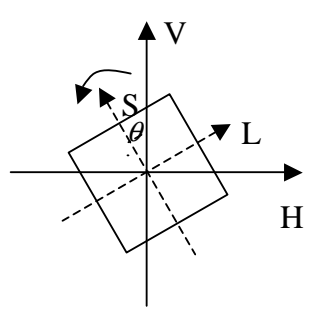

(b)
Fig. 1 (Color online)The rectangle hole arrays. (a) Scanning electron microscope pictures. (b) Rotation direction. S (L) is the axis of short (long) edge of rectangle hole; $\mathrm{H}(\mathrm{V})$ is horizontal (vertical) axis. 
1(b). Transmission spectra of the hole arrays for rotation angle $\theta=0^{\circ}$ and $90^{\circ}$ were given in Fig. 2. There were large difference between the two cases, which was also observed in 18 .

Further, the typical hole array $(100 \mathrm{~nm} \times 300 \mathrm{~nm}$ holes $)$ was rotated anti-clockwise in the plane perpendicular to the illuminating light(see Fig.1 (b)). Transmission efficiencies of $H$ and $V$ photons(702nm wavelength) were measured with rotation angle $\theta=0^{\circ}, 30^{\circ}, 45^{\circ}, 60^{\circ}$, and $90^{\circ}$ respectively, as shown in Fig. 3. They were varied with $\theta$. To explain the results, we gave a simple model. For our sample, photons with $702 \mathrm{~nm}$ wavelength will excite the SPP eigenmodes $(0, \pm 1)$ and $( \pm 1,0)$. Since the SPPs were excited in the directions of long (L) and short (S) edges of rectangle holes, we suspected that this

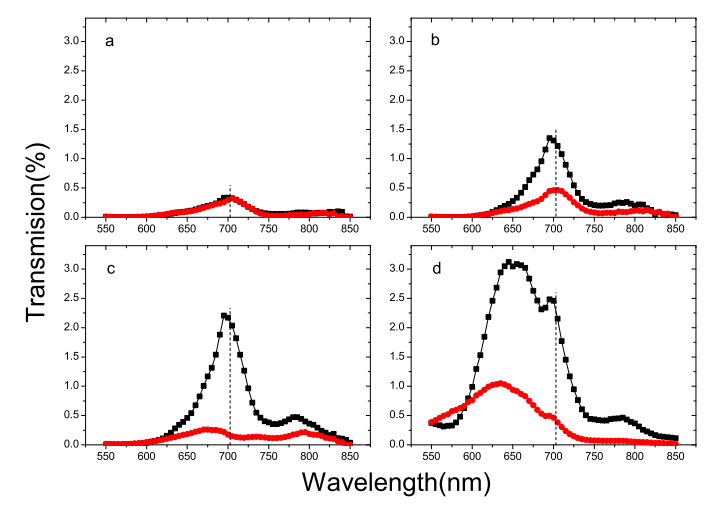

Fig. 2 (Color online)Hole array transmittance as a function of wavelength for rotation angle $\theta=0^{\circ}$ (black square dots) and $90^{\circ}$ (red round dots)(holes for a, b, c, and d are $100 \mathrm{~nm} \times$ $100 \mathrm{~nm}, 100 \mathrm{~nm} \times 150 \mathrm{~nm}, 100 \mathrm{~nm} \times 200 \mathrm{~nm}$, and $100 \mathrm{~nm} \times$ $300 \mathrm{~nm}$ respectively). The dashed vertical lines indicate the wavelength of $702 \mathrm{~nm}$ used in the experiment. two directions were eigenmode-directions for our sample.

The polarization of illuminating light was projected into the two eigenmode-directions to excite SPPs. After that, the two kinds of SPPs transmitted the holes and irritated light with different transmission efficiencies $T_{L}$ and $T_{S}$ respectively. For light whose polarization had an angle $\theta$ with the $S$ direction, the transmission efficiency $T_{\theta}$ will be

$$
T_{\theta}=T_{S} \cos ^{2}(\theta)+T_{L} \sin ^{2}(\theta) .
$$

This equation was also given in the works 20,21]. Due to the unequal values of $T_{L}$ and $T_{S}$, the whole transmission efficiency was varied with angle $\theta$. So if we know the transmission spectra for enginmode-directions (here $\mathrm{L}$ and $\mathrm{S}$ ), we can calculate out the transmission spectra (including the heights and locations of peaks) for any

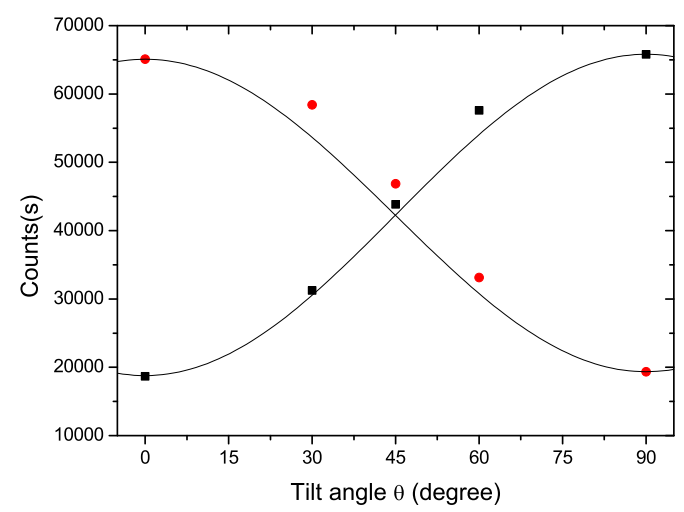

Fig. 3 (Color online)Transmittance as a function rotation angle $\theta$ for photons in $702 \mathrm{~nm}$ wavelength $(100 \mathrm{~nm} \times 300 \mathrm{~nm}$ holes). Red round dots and black square dots are the counts for $V$ and $H$ photons respectively. The lines come from theoretical calculation. 
$\theta$. The theoretical calculations were also given in Fig.

3, which agreed well with the experimental data. The similar results were also observed when the hole arrays $(100 \mathrm{~nm} \times 150 \mathrm{~nm}$ and $100 \mathrm{~nm} \times 200 \mathrm{~nm})$ were used. With this model, the transmission efficiency can be continuously tuned in a certain range.

\subsection{Influence of hole shape on photon polarization}

To investigate the polarization property of the hole array, we used the method of polarization state tomography. Experimental setup was shown in Fig. 4. White light from a stabilized tungsten-halogen source passed though single mode fiber and $4 \mathrm{~nm}$ filter (center wavelength $702 \mathrm{~nm}$ ) to generate $702 \mathrm{~nm}$ wavelength photons. Polarization of input light was controlled by a polarizer, a HWP (half wave plate, 702nm) and a QWP (quarter wave plate, $702 \mathrm{~nm})$. The hole array was set between two lenses of $35 \mathrm{~mm}$ focal length. Symmetrically, a QWP, a HWP and a polarizer were combined to analyze the polarization of transmitted photons. For arbitrary input states, the output states were measured in the four bases: $H, V, 1 / \sqrt{2}(|H\rangle+|V\rangle)$, and $1 / \sqrt{2}(|H\rangle+i|V\rangle)$. With these experimental data, we could get the density matrix of output states, which gave the full polarization characters of transmitted photons. For example, in the case of $\theta=0^{\circ}$, for input state $1 / \sqrt{2}\left(|H\rangle+e^{I * 0.5 \pi}|V\rangle\right)$, four counts $(8943,31079,3623$ and 21760) were recorded when we used the four detection bases. The density ma- trix was calculated as:

$$
\left(\begin{array}{cc}
0.223 & -0.410-0.043 i \\
-0.410+0.043 i & 0.777
\end{array}\right)
$$

which had a fidelity of 0.997 with the pure state $0.472|H\rangle+$ $0.882 e^{I * 0.967 \pi}|V\rangle$. Compared this state with the input state, we found that not only the ratio of $|H\rangle$ and $|V\rangle$ was changed, but also a phase $\varphi=0.467 \pi$ was added between them. The similar phenomenon was also observed when the input state was $1 / \sqrt{2}(|H\rangle+|V\rangle)$ and in this case $\varphi=0.442 \pi$. We also considered the cases for $\theta=30^{\circ}, 45^{\circ}, 60^{\circ}$, and $90^{\circ}$. The experimental density matrices had the fidelities all larger than 0.960 with the theoretical calculations, where $\varphi=(0.462 \pm 0.053) \pi$. It can be seen that the phase $\varphi$ was hardly influenced by the rotation.

To study the dependence of phase $\varphi$ with the hole shape, we performed the same measurements on other hole arrays which were shown in Fig. 1. It was found that $\varphi$ was changed with the aspect ratio of the rectan-

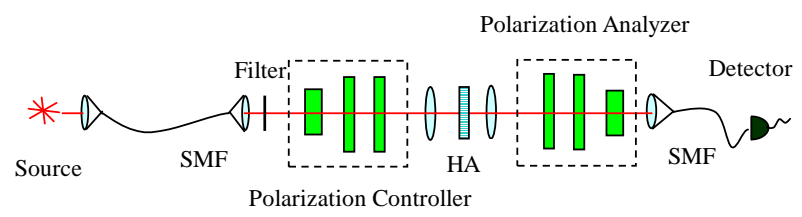

Fig. 4 (Color online)Experimental setup to investigate the polarization property of our rectangle hole array. Polarization of input light was controlled by a polarizer, a HWP and a QWP. The hole array was set between two lenses of $35 \mathrm{~mm}$ focal length. Symmetrically, a QWP, a HWP and a polarizer were combined to analyze the polarization of transmitted photons. 
gle holes. Fig. 5 gave the relation between $\varphi$ and aspect ratio. The phases are $0,(0.227 \pm 0.032) \pi,(0.357 \pm 0.020) \pi$ and $(0.462 \pm 0.053) \pi$ for aspect ratio $1,1.5,2.0$ and 3.0 respectively. As mentioned above, period is another important parameter in the EOT experiments. Since no similar result was observed for hole arrays with symmetrical periods, a special quadrate hole array(see Fig. 1 of [21]) was also investigated to show the influence of the hole period. We found that even the periods were different in two directions, there was no birefringent phenomenon $(\varphi=0)$.

This birefringent phenomenon might be explained with the propagating of SPPs on the metal surface. As we know, the interaction of the incident light with surface plasmon is made allowed by coupling through the grating momentum and obeys conservation of momentum

$$
\vec{k}_{s p}=\vec{k}_{0} \pm i \vec{G}_{x} \pm j \vec{G}_{y}
$$

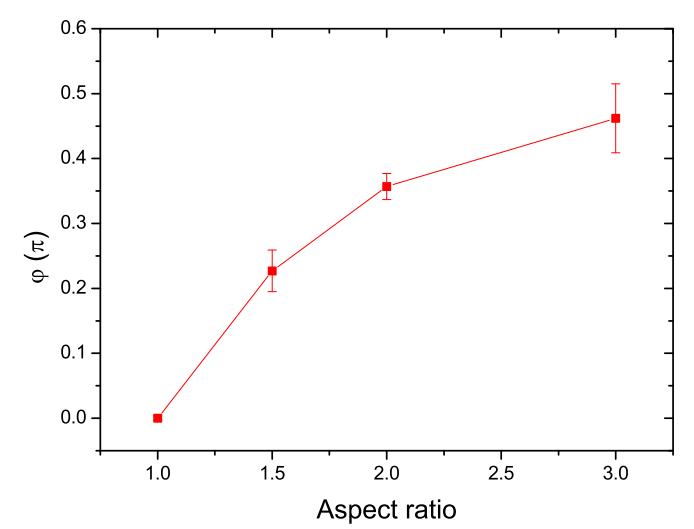

Fig. 5 (Color online)Relation between birefringent phase $\varphi$ and hole shape aspect ratio. $\varphi$ becomes lager when the aspect ratio increases. where $\vec{k}_{s p}$ is the surface plasmon wave vector, $\vec{k}_{0}$ is the component of the incident wave vector that lies in the plane of the sample, $\vec{G}_{x}$ and $\vec{G}_{y}$ are the reciprocal lattice vectors, and i, j are integers. Usually, $G_{x}=G_{y}=2 \pi / d$ for a square lattice, and relation $\vec{k}_{s p} * d=m \pi$ was satisfied, where $m$ was the band index 22$]$. While for our rectangle hole arrays, the length of holes in $L$ direction was changed form $150 \mathrm{~nm}$ to $300 \mathrm{~nm}$, which was not as same as it in $S$ direction. Though $G_{x}=G_{y}=2 \pi / d$ for our rectangle hole array, the time for surface plasmon polariton propagating in the $L$ direction must be influenced by the aspect ratio of hole shape, which could not be same as that in the $S$ direction. A phase difference $\varphi$ was generated between the two directions, leading the birefringent phenomenon. Due to the absorption or scattering of the SPPs and scattering at the hole edges, it is hard to give the accurate value of the phase or the exact relation between the phase and aspect ratio of holes. Even so, $\varphi$ could be controlled by changing the hole shape. As a contrast, there was no birefringent phenomenon observed when the quadrate hole array(see Fig. 1 of [21]) was used. The reason was that phase $G_{x} * d_{x}$ always equal to $G_{y} * d_{y}$, even $G_{x} \neq G_{y}$ for the quadrate hole array.

\section{3 conclusion}

In conclusion, rectangle hole array was explored to study the influence of hole shape on EOT, especially the properties of photon polarization. Because of the unsymmet- 
rical of the hole shape, a birefringent phenomenon was observed. The phase was determined by the hole shape, which gave us a potential method to control this birefringent process. It was also found that the transmission efficiency can be tuned continuously by rotating the hole array. These results might be explained using an intuitional model based on surface plasmon eigenmodes.

This work was funded by the National Fundamental Research Program, National Natural Science Foundation of China (10604052), Program for New Century Excellent Talents in University, the Innovation Funds from Chinese Academy of Sciences, the Program of the Education Department of Anhui Province (Grant No.2006kj074A). Xi-Feng Ren also thanks for the China Postdoctoral Science Foundation (20060400205) and the K. C. Wong Education Foundation, Hong Kong.

\section{References}

1. T.W. Ebbesen, H. J. Lezec, H. F. Ghaemi, T. Thio, and P. A. Wolff, Nature 391, 667 (1998).

2. H. Raether, Surface Plasmons on Smooth and Rough Surfaces and on Gratings, Vol. 111 of Springer Tracts in Modern Physics, Springer, Berlin, (1988).

3. D. E. Grupp, H. J. Lezec, T. W. Ebbesen, K. M. Pellerin, and Tineke Thio, Appl. Phys. Lett. 771569 (2000).

4. M. Moreno, F. J. Garca-Vidal, H. J. Lezec, K. M. Pellerin, T. Thio, J. B. Pendry, and T. W. Ebbesen, Phys. Rev. Lett. 86, 1114 (2001).
5. S. M. Williams, K. R. Rodriguez, S. Teeters-Kennedy, A. D. Stafford, S. R. Bishop, U. K. Lincoln, and J. V. Coe, J. Phys. Chem. B. 108, 11833 (2004).

6. A. G. Brolo, R. Gordon, B. Leathem, and K. L. Kavanagh, Langmuir. 20, 4813 (2004).

7. A. Nahata, R. A. Linke, T. Ishi, and K. Ohashi, Opt. Lett. $28,423(2003)$

8. X. Luo and T. Ishihara, Appl. Phys. Lett. 84, 4780 (2004).

9. S. Shinada, J. Hasijume and F. Koyama, Appl. Phys. Lett. $83,836(2003)$.

10. C. Genet and T. W. Ebbeson, Nature, 445, 39 (2007).

11. J. Elliott, I. I. Smolyaninov, N. I. Zheludev, and A. V. Zayats, Opt. Lett. 29, 1414 (2004).

12. R. Gordon, A. G. Brolo, A. McKinnon, A. Rajora, B. Leathem, and K. L. Kavanagh, Phys. Rev. Lett. 92, 037401 (2004).

13. E. Altewischer, C. Genet, M. P. van Exter, and J. P. Woerdman, Opt. Lett. 30, 90 (2005).

14. X. F. Ren, G. P. Guo, Y. F. Huang, Z. W. Wang, and G. C. Guo, Opt. Lett. 31, 2792, (2006).

15. X. F. Ren, G. P. Guo, Y. F. Huang, C. F. Li, and G. C. Guo, Europhys. Lett. 76, 753 (2006).

16. E. Altewischer, M. P. van Exter and J. P. Woerdman Nature 418304 (2002).

17. S. Fasel, F. Robin, E. Moreno, D. Erni, N. Gisin and H. Zbinden, Phys. Rev. Lett. 94110501 (2005).

18. K. J. Klein Koerkamp, S. Enoch, F. B. Segerink, N. F. van Hulst and L. Kuipers, Phys. Rev. Lett. 92183901 (2004)

19. Zhichao Ruan and Min Qiu, Phys. Rev. Lett. 96233901 (2006). 
20. M. Sarrazin, J. P. Vigneron, Opt. Commun. 24089 (2004) .

21. X. F. Ren, G. P. Guo, Y. F. Huang, Z. W. Wang, and G. C. Guo, Appl. Phys. Lett. 90, 161112 (2007).

22. F. L. Tejeira, S. G. Rodrigo, L. M. Moreno, F. J. G. Vidal, E. Devaux, T. W. Ebbesen, J. R. Krenn, I. P. Radko, S. I.Bozhevolnyi, M. U. Gonzalez, J. C. Weeber, and A. Dereux, Nature Physics 3, 324 (2007). 